\title{
An Application of Approximation Theory to an Error Estimate in Linear Algebra
}

\author{
By Geneva G. Belford* and E. H. Kaufman, Jr.
}

\begin{abstract}
Recently developed theorems characterizing best uniform approximations from a family with a fixed point are shown to be useful in the estimation of errors in computational techniques for solving linear algebraic equations. Specifically, a gap in the proof of a published theorem is filled in.
\end{abstract}

In the literature on computational linear algebra, one increasingly sees references to a paper by Kaniel [1] which gives several very sharp error estimates. To obtain one of these estimates, Kaniel invokes the classical Chebyshev alternation theorem. The context, however, is one in which the classical theorem does not apply. The purpose of this note is to show how the gap in Kaniel's proof may be filled in by applying more general approximation theorems which have recently appeared in the literature.

Kaniel discusses the problem of obtaining an approximate solution to the matrix equation $A x=f$, where $A$ is an $l \times l$ positive definite matrix and the approximate solution $\tilde{x}$ is to lie in the subspace $V$ spanned by the vectors $\left\{f, A f, \ldots, A^{n-1} f\right\}$. A best least-squares solution from $V$ is defined as an element $\tilde{x}=\sum_{i=0}^{n-1} \alpha_{i} A^{i} f$ which minimizes $R \equiv\|A \tilde{x}-f\|_{2}$, where the norm here is the Euclidean or $L_{2}$ norm. Kañiel proceeds to determine an upper bound on the minimal $R$ as follows. First, note that $R$ can also be written in the form $R=\|p(A) f\|_{2}$, where

$$
p(A)=\sum_{i=0}^{n-1} \alpha_{i} A^{i+1}-I, \quad I \text { being the identity matrix. }
$$

Let $H$ be the subspace spanned by $\left\{f, \ldots, A^{n} f\right\}$. Let the $l \times l$ matrix $P$ be the orthogonal projection onto $H$ and define $B$ to be the restriction of $P A$ to $H$. Kaniel then obtains the following theorem.

THEOREM (THEOREM 2.1 OF [1]). Let $p(x)$ be a polynomial of degree at most $n$ and satisfying $\left|p\left(\mu_{i}\right)\right| \leqq \varepsilon$ for $i=1, \ldots, n+1$ (where the $\mu_{i}^{\prime}$ 's are the eigenvalues of $\left.B\right)$. Then

$$
\|p(A) f\|_{2} \leqq \varepsilon\|f\|_{2} \text {. }
$$

To get a tight bound on $R$, Kaniel suggests the construction of a polynomial $p(x)$

Received August 29, 1973.

AMS (MOS) subject classifications (1970). Primary 65F10, 41 A50.

Key words and phrases. Uniform approximation, numerical linear algebra, best approximation, leastsquares solution.

* Work of this author was supported in part by the Advanced Research Projects Agency of the Department of Defense and was monitored by the U. S. Army Research Office-Durham under Contract No. DAHC 04-72-C-0001.

Copyright $\odot 1974$, American Mathematical Society 
which minimizes $\varepsilon \equiv \max _{i}\left|p\left(\mu_{i}\right)\right|$ over the set $P_{n}^{\prime}$ of all polynomials of degree at most $n$ and satisfying the constraint $p(0)=-1$ (cf. (1)). Kaniel correctly states this polynomial to be

$$
p(x)=\frac{-r(x)}{r(0)}, \quad \text { where } r(x)=\sum_{j=1}^{n+1}(-1)^{j} \frac{\prod_{i \neq j}\left(x-\mu_{i}\right)}{\prod_{i \neq j}\left(\mu_{j}-\mu_{i}\right)} .
$$

In spite of the fact that this problem is nonstandard, he quotes the standard linear Chebyshev theorem as justification.

The relevant theory is actually that of approximation with "constraints" as discussed in [2]. Because of the generality of the theorems in that paper, we include here a brief guide to how the theory applies to the case at hand.

Assuming that the eigenvalues are distinct and ordered, $0<\mu_{1}<\mu_{2}<\cdots$ $<\mu_{n+1}$, we are interested in approximation on the interval $\left[0, \mu_{n+1}\right]$. In the terminology of [2], the set $P_{n}^{\prime}$ is $S$-unisolvent of degree $n$ on $\left[0, \mu_{n+1}\right]$. (See Corollary 2 and Theorem 6 of [2].) We may then apply Theorem 7 of [2], with $X=\left[0, \mu_{n+1}\right]$ and $X_{1}=\left\{\mu_{1}, \ldots, \mu_{n+1}\right\}$. Since the constraint point is an endpoint of $X$, the " $S$ alternations" of Theorem 7 become ordinary alternations. We then see that (3) does give the unique minimizing polynomial, the $n+1$ points of the alternant being simply the $\mu_{i}$ 's themselves.

Center of Advanced Computation

University of Illinois at Urbana-Champaign

Urbana, Illinois 61801

Mathematics Department

Central Michigan University

Mount Pleasant, Michigan 48858

1. S. Kaniel, "Estimates for some computational techniques in linear algebra," Math. Comp., v. 20, 1966, pp. 369-378. MR 38 \#2934.

2. E. H. Kaufman, JR. \& G. G. Belford, "A generalization of the varisolvency and unisolvency properties," J. Approximation Theory, v. 7, 1973, pp. 21-35. 